\title{
Philosophiques
}

\section{En collaboration, Le phénomène IXE-13, Québec, P.U.L. («Vie des lettres québécoises, 21 »), 1984.}

\section{Jean-Claude Simard}

Volume 14, numéro 1, printemps 1987

URI : https://id.erudit.org/iderudit/027010ar

DOI : https://doi.org/10.7202/027010ar

Aller au sommaire du numéro

Éditeur(s)

Société de philosophie du Québec

ISSN

0316-2923 (imprimé)

1492-1391 (numérique)

Découvrir la revue

Citer ce compte rendu

Simard, J.-C. (1987). Compte rendu de [En collaboration, Le phénomène IXE-13, Québec, P.U.L. («Vie des lettres québécoises, 21 »), 1984.] Philosophiques, 14(1),

208-213. https://doi.org/10.7202/027010ar d'utilisation que vous pouvez consulter en ligne.

https://apropos.erudit.org/fr/usagers/politique-dutilisation/ 
EN COllaboration, Le phénomène IXE-13, Québec, P.U.L. ( lettres québécoises, $21 »), 1984$.

\section{par Jean-Claude Simard}

Les études philosophiques ont leurs points aveugles. Certains phénomènes importants, plus propres au $\mathrm{XX}^{\mathrm{e}}$ siècle, ont peu retenu l'attention des penseurs dits professionnels. Citons au hasard, pour s'en tenir au domaine culturel, le cinéma, la photographie, le roman policier et la science-fiction. Si l'on excepte quelques cas isolés (Barthes et Sonntag pour la photographie, Benjamin et Deleuze - depuis peu - pour le cinéma, Butor pour la science-fiction et Bloch, enfin, pour le policier), ces domaines sont en général restés en marge de la recherche dite savante.

La même situation prévaut mutatis mutandis dans certains domaines des études littéraires ou sémiotiques. La littérature de masse, par exemple et particulièrement celle du Québec - a, plus souvent qu'autrement, été observée de loin : production industrielle, médiocrité esthétique, rentabilité à courte vue, affirme-t-on volontiers à son propos. Depuis quelque temps, cependant, le département des littératures de l'Université Laval a donné naissance à un important projet de recherche sur la littérature québécoise en fascicules : LIQUEFASC. C'est dans un tel cadre qu'ici réunis, six auteurs en quête de personnages ont étudié «le phénomène IXE-13». Car il faut bien parler en effet d'un authentique phénomène. Cette série de romansfeuilletons, intitulée Les aventures étranges de IXE-13, l'as des espions canadiens, a paru pendant près de vingt ans, de 1947 à 1966. Neuf cent trente-quatre fascicules d'environ trente-deux pages chacun, à un rythme approximativement hebdomadaire, à raison de quelque trente mille ventes par numéro: près de vingt et un million d'exemplaires écoulés! Au moment où l'auteur, Pierre Saurel (pseudonyme de Pierre Daignault), vient de lancer un nouveau personnage appelé «le manchot», il est particulièrement heureux que l'on se penche enfin sérieusement sur IXE-13, ce curieux numéro.

L'ouvrage se présente un peu comme un bilan de recherche et il aborde l'analyse sous au moins quatre points de vue différents : socio-économique, narratologique, psychanalytique et idéologique. Le tout est encadré d'une introduction et d'une conclusion visant à donner à l'ensemble, par-delà l'inévitable tension qu'engendre cette disparité d'approches, une unité minimale de conception.

La première étude, due à Vincent Nadeau et Michel René, s'intitule: «Histoire d'une littérature industrielle ». On y fait connaissance avec Pierre Daignault, lui-même personnage haut en couleurs, puisque comédien et acteur, animateur et folkloriste, sans oublier bien sûr scripteur et auteur. On ne s'empressera pas de dénoncer aussitôt, ce lisant, le vieux schéma éculé de l'histoire littéraire: l'homme (chapitre 1) et l'œuvre (chapitres suivants) puisque si cette longue première section est en effet largement biographique, 
elle est encore et surtout l'occasion d'assister à l'élaboration d'un «produit industriel de masse» (p. 328). De sorte que les informations sur la vie de notre auteur sont constamment articulées aux conditions de production et de circulation de l'œuvre. On nous y montre comment une mise en marché fort efficace, l'absence de concurrents sérieux et surtout le processus d'alphabétisation et de scolarisation des adolescentes et adolescents québécois des années cinquante, ont pu transformer une petite maison en une vaste entreprise dont la «marchandise» IXE-13 constituait très certainement le pivot central.

L'entrée en matière de Nadeau et René est certes la bienvenue si l'on prétend asseoir ainsi les soubassements nécessaires de l'ouvrage, c'est-à-dire l'infrastructure économique de ce type de littérature dont c'est, nous l'avons mentionné, l'un des aspects principaux. Leur étude soulève cependant deux difficultés. Le foisonnement généreux des dates, noms, chiffres, etc., qui se superposent longuement et s'entrecroisent sur près de soixante pages devient vite fastidieux et, partant, lassant. Lacune d'ordre esthétique sans doute et somme toute assez inoffensive, une grisaille inattendue n'étant parfois que le repoussoir indésiré du patient labeur. Il n'en va pas de même cependant de la deuxième difficulté, plus sérieuse, qui a plutôt trait à la méthodologie. Les renseignements dans le domaine de la littérature de masse - ce qu'on est souvent convenu d'appeler la paralittérature, bien que les deux termes ne se recouvrent pas exactement - sont habituellement nombreux, mais de piètre qualité. D'où le fait qu'il faille à l'occasion recourir à des documents dont la valeur est discutable, comme c'est ici le cas. Lacune autrement plus lourde de conséquence, car citer sur un même pied, et parfois à quelques lignes de distance, le Dictionnaire des oewvres littéraires $d u$ Québec, d'une part, et Photo-Journal, Police-Journal ou Le Journal de Montréal, d'autre part, pose, on l'admettra sans peine, certains problèmes délicats ${ }^{1}$. Il aurait été souhaitable, me semble-t-il, d'établir pour cette première étude une brève évaluation critique des sources disponibles.

Quoi qu'il en soit, reste qu'il était nécessaire d'analyser d'abord, comme on le dit fort à propos, les «facteurs de production» (p. 67) et que ce chapitre, malgré les quelques lacunes dont il a été fait état, est dans l'ensemble assez solide et surtout, fort instructif.

La deuxième étude, «Les structures du roman d'espionnage », introduit l'angle narratologique qui sera encore exploité par la suite. L'auteur, Guy Bouchard, y revient à ses anciennes amours puisque sa thèse de doctorat

1. Exemple parmi d'autres de ces problèmes: "Attiré par le roman policier? Je ne l'ai jamais vraiment été de façon spéciale" (Pierre Daignault, in Photo-Journal, 2-11-1970. "J'ai surtout le besoin d'écrire des romans policiers, c'est ma spécialité " ("propos du mois de décembre 1977 » (sic)). Ces deux affirmations, citées à cinq pages d'intervalle $(57,62)$, s'accordent plutôt mal et mettent en cause la validité de leur source respective. Nos deux auteurs, s'ils en ont pris conscience, n'en ont en tout cas rien laissé paraître. 
(1971) portait précisément sur cette question. Il s'emploie ici «à dégager les structures du contenu à l'œuvre dans la série IXE-13» (p. 73).

Il s'agit d'une analyse habile, longue - plus de cent pages - et ambitieuse où, sur la base d'un point de vue synchronique, on espère parvenir à « un certain nombre de propositions théoriques qui (...) seraient applicables au récit d'espionnage comme tel, sinon à toute œuvre romanesque » (p. 73). On peut reconnaître dans l'appareil conceptuel auquel il est fait appel une assimilation efficace de nombreux travaux antérieurs: pour l'approche d'ensemble, la sémiotique de Greimas (Sémantique structurale), pour l'obtention du corpus, le modèle donné par Propp (Morpbologie du conte), pour l'énonciation des propositions narratives, les thèses actantielles de Souriau (Les deux cent mille situations dramatiques), pour le découpage textuel, les lexies utilisées à l'occasion par Barthes $(S / Z)$, et enfin certains éléments des analyses récentes sur le récit d'espionnage, telle celle sur James Bond par Umberto Eco.

Bouchard utilise, comme hypothèse de départ, un scénario schématique, «modelé sur le carré sémiotique ( $\mathrm{p}$. 75). Aménagé sous ses divers chefs, il permet d'obtenir une première gamme de procès narratifs fondamentaux : capture, trahison, mainmise, assassinat, neutralisation et sabotage. Cette formalisation amène une confrontation initiale du corpus, réparti proportionnellement aux classes dégagées. Considérant ensuite comme sujets et prédicats respectifs les rôles des personnages - qu'il appelle «indices»- et leurs aspects (actions, éléments psychologiques, etc.), l'auteur parvient à des « propositions narratives » propres à les caractériser. Le personnage se transforme ainsi en «signifié global» d'un roman luimême considéré comme "signe ». Bouchard procède alors, via la technique des lexies, à la construction d'une structure syntagmatique, en utilisant comme récit-témoin Le repaire de la mort, premier de la série ${ }^{2}$. Puis, faisant montre d'une rigueur exemplaire, il analyse le tout sous l'angle double de la logique et de la chronologie pour aboutir, à la suite de réductions successives, à une structure globale qui semble propre à subsumer les possibilités narratives de la série IXE-13 tout entière. Il ne reste enfin qu'à comparer les scénarios majeurs obtenus à ceux des romans de Fleming (Bond) ou de Bruce (OSS 117) pour observer une même logique à l'œuvre. La conclusion s'impose d'elle-même: "Cette formule n'est pas propre à la série IXE-13 (...). La structure est typique, l'investissement est folklorique» (p. 177).

Cette deuxième étude aboutit ainsi à dégager deux registres sur lesquels vont s'appuyer les textes postérieurs. Qui dit folkloricité dit aussi lieu d'inscription possible pour une idéologie, comme le montrera la cinquième section de l'ouvrage, sur laquelle nous reviendrons. D'autre part, qui parle de structure globale parle aussi de programme narratif. C'est ce que s'applique à démontrer Louise Milot dans le chapitre qui suit : "La défaite des femmes ».

2. Les raisons d'un tel choix ne sont pas indiquées. Peut-être l'auteur considère-t-il que tout autre choix eût abouti aux mêmes conclusions? 
En théorie, le héros d'espionnage n'a pas droit à une vie sentimentale, encore moins à un lien a moureux stable. Or, non seulement IXE-13 aime-t-il Gisèle Tubœuf, mais il finit même par l'épouser! L'habileté de Louise Milot consistera à faire la preuve que, malgré les apparences, une telle situation n'est nullement une entorse à la «logique contraignante du récit» (p. 182), mais vient en fait confirmer la primauté indiscutable du programme narratif d'espionnage sur celui de la vie amoureuse. L'auteure analyse le cas de trois femmes jouant un rôle majeur dans la vie du héros, pour aboutir toujours à la même conclusion. Ainsi Gisèle Tubœuf, espionne confirmée au début de la série, sans aucun doute l'égale de IXE-13 lui-même, est peu à peu reléguée après leur union à une vie débilitante : impuissante, inactive et « clouée sur un lit d'hôpital» (p. 223). Cette "occultation progressive » constitue une preuve parmi d'autres de l'hypostasie de la trame narrative de l'espionnage et réaffirme le rôle subordonné des femmes, lesquelles, en un tel monde, tendent à devenir petit à petit des êtres «de service» (p. 226). Elle vient aussi confirmer indirectement l'optique structurale en général (et par conséquent les conclusions antérieures de Guy Bouchard) : si l'existence d'un agent secret amoureux constitue un «malaise narratif» qu'il faut graduellement réduire, c'est que la présence d'une structure implique un ordre global et donc une résistance à son contraire. Or la série ne dispose, pour pallier au désordre, que de capacités d'élasticité limitées. Elie consacrera toujours «la défaite des femmes».

Dans le texte qui suit, «La structure psychanalytique», Claude-Marie Gagnon vise, pour sa part, «à l'établissement d'un mythe psychanalytique particulier à une production de fiction» (p. 229). S'éloignant sensiblement de la psychocritique de Charles Mauron dont il fait par ailleurs un large usage, Gagnon cherche à lui adjoindre les acquis récents du structuralisme et de la sociocritique. Liant la série à une « quête d'identité sexuelle » (p. 239), il table sur l'ambivalence d'IXE-13 face au mariage, déjà exploitée dans le précédent chapitre, sur sa relation d'amour-haine avec ses supérieurs ainsi que sur son intense camaraderie, pour dégager peu à peu la structure oedipienne de ses aventures. Selon lui, le personnage d'IXE-13 ferait montre d' « homosexualité latente » et d'un refus d'assumer la «vision génitale du monde » déterminant l'entrée dans l'âge adulte. Ce qui dessine la structure psychologique propre à l'adolescence. On rejoint par ce biais les constats de la première étude touchant le processus de scolarisation des années cinquante et on explique du même coup les raisons d'un extraordinaire succès de librairie.

Le chapitre, plutôt concluant jusqu'à ce point, eût pu s'achever sur cette note heureuse. Mais l'auteur se lance alors dans d'audacieuses considérations qui, quoiqu'éminemment riches, n'en reposent pas moins sur des modes d'articulations assez problématiques. Lorsqu'on compare les aventures d'IXE-13 à d'autres séries réalisées à la même époque pour en arriver à conclure que le "roman d'espionnage est aussi le roman de la guerre froide » (p. 274), on se meut encore en terrain stable et on propose une hypothèse féconde. Quand cependant, pour expliciter plus avant l'impact phénoménal d'IXE-13, on aboutit à une vaste comparaison psycho-sociale où le Québec, à 
la veille de la Révolution tranquille, serait une société adolescente sur le point de subir une "mutation» susceptible de l'amener à un âge "plus adulte" (p. 281), on quitte rapidement la terre ferme. Cette métaphore biogénétique, qui possède l'attrait des choses vieilles comme le monde, soulève aussi toute la question de l'explication causale, problème non moins vénérable. Disons pour faire court que, nonobstant son pouvoir de suggestion, je ne crois pas que ce dernier développement entraîne - il s'en faut de beaucoup l'adhésion ferme.

De là, on passe à la cinquième et dernière étude, «L'idéologique dans le texte", due à Denis Saint-Jacques, lequel signe aussi l'introduction et la conclusion. Cette analyse reprend où avait laissé la précédente et poursuit la réflexion sur la contribution de la série IXE-13 à l'histoire du Québec. S'appuyant sur un essai de Marcel Rioux qui distinguait, à l'œuvre durant cette époque, la marque de trois grandes idéologies récurrentes (de conservation, de rattrapage et de développement/participation), SaintJacques montre comment l'œuvre de Pierre Daignault s'inscrit indéniablement dans la mouvance de la seconde.

IXE-13 est le héros d'un désir tout fictif de participation militaire aux activités contemporaines de ce qu'il désigne lui-même du nom de «monde libre », héros dynamique et positif qui n'a plus rien à voir avec le défenseur crispé et sans cesse humilié des valeurs d'autrefois (p. 296).

L'œuvre échappe donc globalement à un idéal de conservation sous la bannière duquel s'abritaient ultimement, effort de maintien tout entier intégriste, la langue, la charrue et la croix. Mieux même, si l'on considère le second axe narratif identifié par Milot, c'est-à-dire le rôle éminent qu'y jouent un temps les femmes, IXE-13 relève peut-être encore de la seconde idéologie, mais annonce aussi et surtout, particulièrement avant que cet axe n'ait été progressivement amoindri et finalement écarté au profit du programme narratif dominant, l'idéologie de développement/participation des années 1960-70. Ainsi s'éclaire, par le biais d'une double appartenance manifeste, la «fonction sociale d'un tel texte » (p. 285) et « la place de ce récit dans son écosystème idéologique 》 (p. 289).

Quand Pierre Elliott Trudeau et Jean Lesage auront enfin rattrapé Pierre Saurel et ses lecteurs, la Révolution tranquille pourra avoir lieu tout démocratiquement (p. 313).

Les conséquences de cette contribution de Saint-Jacques ainsi que de la conclusion qui la prolonge sont intéressantes à plus d'un titre. D'abord, en développant les deux études narratologiques antérieures grâce à une exploration du contenu et de l'expression, elle leur apporte un complément indispensable. Ensuite, en drainant dans son large cours certaines alluvions déposées par les approches socio-économique et psychanalytique, elle permet d'unifier commodément et sans artifice excessif l'ensemble de l'ouvrage. Enfin - et ce n'est certes pas le moindre de ses nombreux mérites - elle met sérieusement en cause une situation qui perdure depuis trop 
longtemps déjà : le privilège de la culture dite savante sur la culture dite de masse. Dans le cas précis qui nous occupe, la

culture de masse, dans ce qu'elle a de plus frustre et de choquant pour les esprits fins et évolués, aura indiqué dans l'imaginaire social la voie de l'avenir à une culture savante trop prête à rendre le duplessisme responsable de toutes ses contradictions (p. 313)

Cette surprenante conclusion montre à quel point s'imposait une lecture approfondie et multidisciplinaire de Pierre Daignault. Quant à ses résultats, ils devraient servir d'avertissement et pour les études littéraires et pour la philosophie. Comme eût pu le prétendre le dicton populaire : il n'y a pas de sots sujets, il n'y a que des préjugés. N'est-ce pas ce qu'affirmaient récemment encore à leur façon Marc Chabot et André Vidricaire? «Philosopher aujourd'hui, c'est (s')ouvrir (...) à tous les objets (...) il n'y a plus pour les philosophes d'objet impropre pour la pensée » 3 .

Souhaitons donc que cet excellent ouvrage constitue un pas appréciable en direction d'une telle compréhension. Peut-être en ce cas pourra-t-on voir se résorber quelque peu cette déplorable tendance qui prétend, sur la paralittérature (ou la paraphilosophie), apposer le label : parents pauvres de l'esprit? Alors le personnage, se faisant justice comme à son habitude, aura en plus contribué à dédouaner son auteur. Ce qui n'aura certes pas été, comme l'affirme plaisamment Guy Bouchard dans un autre contexte, la moins étrange des aventures de l'agent IXE-13, l'as des espions canadiens.

Département de philosophie, Collège de Rimouski. 